\title{
Pharmacokinetics study of a supersaturatable self-microemulsifying drug delivery system for ellagic acid by UHPLC-Q-TOF-MS
}

\author{
JINGLONG WANG ${ }^{1, a}$ \\ LIQUN FAN ${ }^{1, a}$ \\ LIHUA ZHANG ${ }^{1}$ \\ DANDAN ZHENG ${ }^{1, *}$ \\ YINGZI WANG ${ }^{2}$ \\ XIAO SUN ${ }^{3}$ \\ YONGHUI JI \\ ${ }^{1}$ College of Food Sciences and Pharma- \\ ceutical Engineering, Zaozhuang \\ University, Zaozhuang 277160, China \\ ${ }^{2}$ School of Chinese Materia Medica, \\ Beijing University of Traditional Chinese \\ Medicine, Beijing 100029, China \\ ${ }^{3}$ School of Pharmaceutical Sciences \\ Shandong University, Jinan 250012 \\ China
}

Accepted September 22, 2020

Published online November 11, 2020

\begin{abstract}
To evaluate the bioavailability of ellagic acid loaded supersaturatable self-microemulsifying drug delivery system (S-SMEDDS), its pharmacokinetic properties were studied in rats with an ultra-high performance liquid chromatography-quadrupole time-of-flight tandem mass spectrometry. The plasma samples were treated by solid-phase extraction method, and gallic acid was used as the internal standard when determining the concentration of ellagic acid. Results showed that the established analytical method was sensitive and accurate, which is applicable to the pharmacokinetic study of ellagic acid. The drug was found to be absorbed rapidly in vivo, and the plasma concentration-time curve showed double peaks, indicating that ellagic acid were reabsorbed by entero-hepatic circulation after oral administration. Compared with ellagic acid suspension, the apparent clearance of ellagic acid-loaded S-SMEDDS and SMEDDS reduced significantly, and the $A U C_{0 \sim \mathrm{t}}$ of them were 4.7 and 5.8-fold increase, respectively. Therefore, the bioavailability of ellagic acid-loaded S-SMEDDS was higher than that of the suspension and SMEDDS.
\end{abstract}

Keywords: ellagic acid, supersaturatable self-microemulsion, pharmacokinetics, UHPLC-Q-TOF-MS

Supersaturatable self-microemulsifying drug delivery system (S-SMEDDS) is an extension of the self-microemulsifying drug delivery system (SMEDDS), which can inhibit the precipitation and improve the solubility of water-insoluble drugs by adding a precipitation inhibitor into the system (1). In recent years, S-SMEDDS has been used in the oral delivery of many hydrophobic active compounds extracted from herbs or Traditional Chinese Medicine (TCM), increasing the oral bioavailability about 7-fold more than the single drug $(2,3)$. Ellagic acid (EA), a natural active ingredient, is isolated from the extraction of pomegranates, black raspberries, raspberries, strawberries, walnuts or almonds, et al. (4). However, the water solubility of EA is about $0.01 \mathrm{mg} \mathrm{mL}^{-1}$ at $\mathrm{pH} 1.2$ and $0.02 \mathrm{mg} \mathrm{mL}^{-1}$ at

\footnotetext{
*Correspondence; e-mail: zddsdu@163.com

${ }^{\mathrm{a}}$ These authors contributed equally to this work.
} 
$\mathrm{pH}$ 6.8. EA shows rapid absorption, distribution and elimination after oral administration at a dose of $85.3 \mathrm{mg} \mathrm{kg}^{-1}$ in rats, and it may be metabolized by intestinal micro-organisms (5). More than $50 \%$ of EA is bound to plasma proteins, and ionized EA occurs in the stomach and intestine (6). Yan and Yin (7) have found that EA is poorly absorbed after oral administration (50 mg kg-1 in rats), and the pharmacokinetic profile fits the two-compartment model. Its abundant pharmacological activities might be limited by undesirable solubility and bioavailability. S-SMEDDS could be considered as an oral delivery system for ellagic acid to improve its bioavailability and achieve a relatively slower and more sufficient absorption.

Recently, liquid chromatography tandem mass spectrometry (LC-MS/MS) has been widely used for the quantification of pharmaceutical preparations, TCM, biological drugs, et al. $(7,8)$. The common methods used for the treatment of plasma samples include protein precipitation, liquid-liquid extraction and solid-phase extraction (SPE) method (9). Based on the theory of liquid-solid chromatography, the SPE method can adsorb and elute samples selectively, which helps improve the recovery of biological samples $(9,10)$. In this study, a specific and reliable ultra-high performance liquid chromatography-quadrupole time-of-flight tandem mass spectrometry (UHPLC-Q-TOF-MS) method is established to study the pharmacokinetics of EA-S-SMEDDS in rats. It is the first time to evaluate the oral bioavailability of EA-S-SMEDDS in vivo.

\section{EXPERIMENTAL}

\section{Reagents}

Ellagic acid (HPLC $\geq 98 \%$ ) was purchased from Shanghai Yuanye Biological Co. Ltd. (Shanghai, China). Ethyl oleate, Tween 80 and polyvinylpyrrolidone (PVP) K30 were obtained from Hunan Er-Kang Pharmaceutical Co. Ltd. (Changsha, China). Methanol, acetonitrile (Merck, Germany) and other used solvents were of chromatographic grade.

\section{Preparation of EA-S-SMEDDS}

EA-S-SMEDDS was prepared by the previously described method (11). Briefly, the surfactant and co-surfactant (67.5\% Tween 80 and $22.5 \%$ PEG 400) were uniformly mixed with a magnetic stirrer at room temperature. Then, the oil phase (10 \% ethyl oleate) was added into the mixture to prepare a blank SMEDDS. After dissolving precipitation inhibitors (0.5\% PVP K30) into the blank SMEDDS, $4 \mathrm{mg} \mathrm{g}^{-1}$ ellagic acid was mixed by vigorous vortexing until a transparent solution was reached, and then the EA-S-SMEDDS was obtained. Meanwhile, EA-SMEDDS was prepared by the same method without the use of precipitation inhibitors. Additionally, the morphologies of EA-S-SMEDDS and EA-SMEDDS were investigated by the HT7700 transmission electron microscope (TEM, Hitachi, Japan), and the average particle size of the emulsions was measured using the Mastersizer 2000 particle size analyzer (Malvern Instruments Ltd., UK). The morphology of emulsified EA-S-SEDDS and EA-SMEDDS presented a round shape and non-aggregated, displaying a narrow particle size distribution with the average size of about $45 \mathrm{~nm}$. 


\section{UHPLC-Q-TOF-MS conditions}

To analyze the content of ellagic acid in rat plasma, an Agilent 1290 Infinity liquid chromatography system, equipped with Agilent 6530 Q-TOF mass spectrometric system (Agilent Technologies, USA) was used in this study.

An Infinitylab EC-C ${ }_{18}$ column $(2.10 \mathrm{~mm} \times 100 \mathrm{~mm}, 2.7 \mu \mathrm{m})$ (Agilent, USA) with a gradient elution was applied to conduct the separation. The sample injection volume was $20 \mu \mathrm{L}$ and the flow rate was $0.2 \mathrm{~mL} \mathrm{~min}{ }^{-1}$. The column temperature was set at $30{ }^{\circ} \mathrm{C}$ and the UVdetection wavelength was $254 \mathrm{~nm}$. The mobile phase was composed of acetonitrile (A) and $0.1 \%$ formic acid containing $10 \mathrm{mmol} \mathrm{L}^{-1}$ ammonium acetate (B). The solvent gradient program of UHPLC analysis is shown in Table I.

The MS detection was performed on a Q-TOF mass spectrometer with ESI source in the negative ionization mode. The optimal MS parameters were as follows: mass spectrum scanning range: $m / z$ 100 1200; nebulizer gas (nitrogen) temperature: $350{ }^{\circ} \mathrm{C}$; nitrogen flow: $11 \mathrm{~L}$ $\mathrm{min}^{-1}$; nebulizer gas pressure: $50 \mathrm{psi}$; capillary voltage: $4000 \mathrm{~V}$. The selected ionization pairs $(\mathrm{m} / \mathrm{z})$ of ellagic acid and gallic acid were $301.2\left([\mathrm{M}-\mathrm{H}]^{-}\right)$and $169.1\left([\mathrm{M}-\mathrm{H}]^{-}\right)$, respectively.

Table I. Solvent gradient program of UHPLC analysis of ellagic acid

\begin{tabular}{ccc}
\hline Time $(\min )$ & A (\%) & B (\%) \\
\hline 0 & 5 & 95 \\
3 & 10 & 90 \\
6 & 80 & 20 \\
9 & 100 & 0 \\
11 & 5 & 95 \\
16 & 5 & 95 \\
\hline
\end{tabular}

The mobile phase: A - acetonitrile, B - $0.1 \%$ formic acid containing $10 \mathrm{mmol} \mathrm{L}-1$ ammonium acetate

\section{SPE method for the extraction of plasma samples}

The $\mathrm{C}_{18}$ SPE cartridges ( $1 \mathrm{~mL}, 30 \mathrm{mg}$, Beijing Dikema Technology Co., Ltd., China) was activated by $1 \mathrm{~mL}$ of methanol and equilibrated by $2 \mathrm{~mL}$ of deionized water. The rat plasma $\left(200 \mu \mathrm{L}\right.$ ) was spiked with $100 \mu \mathrm{L}$ of gallic acid (internal standard, $100 \mathrm{ng} \mathrm{mL}^{-1}$ ) using a vortex for $30 \mathrm{~s}$. The mixed solution was applied to the pre-activated SPE cartridges. Then the cartridges were washed with $2 \mathrm{~mL}$ of deionized water to remove the endogenous impurities in plasma. Finally, $1 \mathrm{~mL}$ of methanol/0.1 \% formic acid $(9: 1, V / V)$ was used to wash the SPE cartridges, and $1 \mathrm{~mL}$ eluents were collected and stored at $-20^{\circ} \mathrm{C}$ before the analysis.

\section{Preparation of standard solution}

The $100 \mu \mathrm{g} \mathrm{mL} \mathrm{m}^{-1}$ stock solution of ellagic acid was diluted to various concentrations with acetonitrile/ $0.1 \%$ formic acid $(1: 9, V / V)$ to obtain the working standard solutions. 
Then, $100 \mu \mathrm{L}$ of the working standard solution was mixed with $100 \mathrm{ng} \mathrm{mL}^{-1}$ internal standard solution (gallic acid) and $200 \mu \mathrm{L}$ of blank rat plasma, followed by treating with the above-mentioned SPE method to prepare the calibration standard solutions $(5,10,40,80$, 160,640 and $800 \mathrm{ng} \mathrm{mL}^{-1}$ ).

\section{Method validation}

The specificity of the method for determining the concentration of ellagic acid in rat plasma was investigated by preparing and analyzing the blank plasma, blank plasma with ellagic acid working standard solution and drug-containing plasma. The drug-containing plasma of rats was collected at $1 \mathrm{~h}$ after oral administration of $40 \mathrm{mg} \mathrm{kg}^{-1}$ EA-S-SMEDDS. The linearity of ellagic acid was analyzed with the peak area ratio (ellagic acid/gallic acid) as the ordinate and the concentration of ellagic acid as the abscissa.

A proper volume of low, medium and high concentration of ellagic acid working standard solution $\left(15,160\right.$ and $\left.640 \mathrm{ng} \mathrm{mL}^{-1}\right)$ and internal standard solution, were added into $200 \mu \mathrm{L}$ of blank plasma. And the hybrid solution was treated according to the method of the 'SPE method for the extraction of plasma samples' to prepare the standard plasma samples (quality control samples).

To investigate the precision of the determination method, the standard plasma samples were measured 5 times in 24 hours to calculate the intra-day precision, and tested by the same method for 5 consecutive days to determine the inter-day precision. The recovery rate of the samples was performed by comparing the peak area of extracted quality control samples with the peak area of extracted blank plasma spiked with standards at the same amount. The matrix effect was evaluated by the ratio of peak areas of the plasma sample (the extracted blank plasma spiked with analytes) versus the working standard solution at the concentration of 15, 160 and $640 \mathrm{ng} \mathrm{mL}^{-1}$, respectively. The blank matrix samples were prepared from 6 different groups of rats, with each group containing 4 individual animals.

The short-term stability of the plasma samples was investigated after preserving the standard plasma samples at $4{ }^{\circ} \mathrm{C}$ for $24 \mathrm{~h}$ and analyzing the samples before and after treatment by the SPE method. The long-term stability was carried out similarly by preserving the three standard plasma samples at $-80^{\circ} \mathrm{C}$ for 2 weeks before being treated by the SPE method. To examine the freeze-thaw stability, the standard plasma samples were stored at $4{ }^{\circ} \mathrm{C}$ for 24 hours and thawed at room temperature. The freeze-thaw operation was repeated for three times.

\section{Animals and pharmacokinetic study}

Twelve healthy male Sprague-Dawley rats (200 \pm 20 g), obtained from Jinan Pengyue Experimental Animal Breeding Co. Ltd. (Jinan, China), were randomly divided into three groups and adaptively fed for one week prior to the experiments. After being kept under fasting with free access to water overnight, the animals were orally administrated with EAS-SMEDDS, EA-SMEDDS and EA suspension $\left(4 \mathrm{mg} \mathrm{mL}^{-1}\right.$ EA dissolved into $0.5 \%$ CMC-Na solution) at a single dose of $40 \mathrm{mg} \mathrm{kg}^{-1}$. About $0.5 \mathrm{~mL}$ blood sample was obtained from rat orbital venous plexus at $0.083,0.167,0.333,0.667,1,2,4,6,8,12$ and $24 \mathrm{~h}$. Then the blood samples were centrifuged for $15 \mathrm{~min}$ at $6262 \mathrm{~g}$. The supernatant plasma was collected and stored at $-80{ }^{\circ} \mathrm{C}$ until analysis. The animal care and all the handling followed the institutional guidelines of the Animal Care and Use Committee of Shandong University. 


\section{Data analysis}

GraphPad Prism 7 software (GraphPad Software, USA) was applied for the statistical analysis (Student's $t$-test). The pharmacokinetic parameters were analyzed by DAS 2.0 (BioGuider Co., China). Values of $p<0.05$ were considered to be statistically significant.

\section{RESULTS AND DISCUSSION}

\section{Method validation}

Fig. 1. displays the chromatograms of ellagic acid and gallic acid. It shows that ellagic acid and the internal standard could be determined in 20 min without any interference from the endogenous substances in rat plasma. The calibration curve for the determination of EA was linear within $5 \sim 800 \mathrm{ng} \mathrm{mL}^{-1}\left(R^{2}=0.9989\right)$. And the regression equation was $y=0.0267 x-0.1851$, whereas $y$ meant the ratio of the peak area of ellagic acid and gallic acid, and $x$ meant the concentration of ellagic acid in rat plasma.

The intra- and inter-day precision for ellagic acid at different concentrations were in the range of $2.54 \sim 4.92 \%$ and $3.78 \sim 6.04 \%$ (relative standard deviation, $R S D$ ), respectively. The extraction recoveries of ellagic acid ranged from 88.11 to $92.34 \%(R S D<6.22 \%)$. And the matrix effect values of ellagic acid at low, medium and high concentrations were 89.21, 90.99 and $90.73 \%$, respectively ( $R S D<7.10 \%$ ). These results revealed that the content determination method for ellagic acid in rat plasma was reproducible with a negligible matrix effect.

The results of stability displayed that ellagic acid in plasma samples remained unchanged after $24 \mathrm{~h}$ at $4{ }^{\circ} \mathrm{C}$ with relative errors $(R E)$ lower than $8.28 \%$. The $R E$ values ranged from -7.33 to $-10.89 \%$ for the long term freezer at $-80{ }^{\circ} \mathrm{C}$ for two weeks, and from -6.85 to $-9.10 \%$ after three freeze-thaw cycles. All these results meet the requirements for analyzing the biological samples.

\section{Pharmacokinetics study}

All the plasma samples were collected at predetermined time points and treated before the determination. The concentration-time curve of ellagic acid exhibited an initial upward and double peaks (Fig. 2), which indicated a rapid absorption of the drug after oral administration, and might be reabsorbed through enterohepatic circulation (12). In addition, the drug concentration-time curves of EA-SMEDDS and EA-S-SMEDDS at each time point were higher compared with EA suspension.

The mean pharmacokinetic parameters for the EA suspension, EA-SMEDDS and EAS-SMEDDS are displayed in Table II. The peak time was within $1 \mathrm{~h}$, and the mean residence time $(M R T)$ was $4.653 \mathrm{~h}$, which suggests that ellagic acid could be absorbed and eliminated quickly. Compared with the EA suspension, the MRT of EA-SMEDDS and EA-SSMEDDS were longer and the clearance was significantly reduced. The increase in $T_{\max }$ and MRT might due to the slower release rate of EA from the inner phase of the microemulsion, thereby prolonging and delaying the process of penetration and distribution of EA into tissues and into the circulation (13).

The $A U C_{0 \sim \infty}$ of EA-SMEDDS and EA-S-SMEDDS were 5.0 and 6.7 times higher than that of EA suspension. The two emulsion formulations also exhibited about a 3-fold 
J. Wang et al.: Pharmacokinetics study of a supersaturatable self-microemulsifying drug delivery system for ellagic acid by UHPLC-QTOF-MS, Acta Pharm. 71 (2021) 679-687.

a)
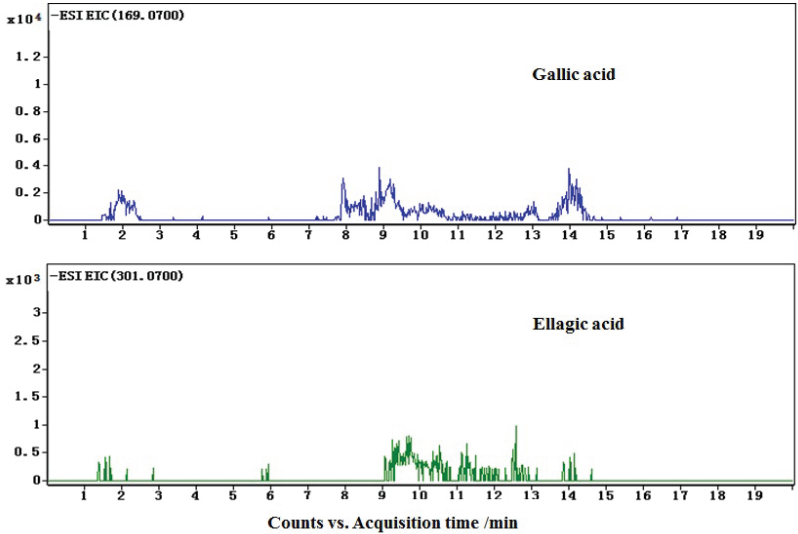

b)
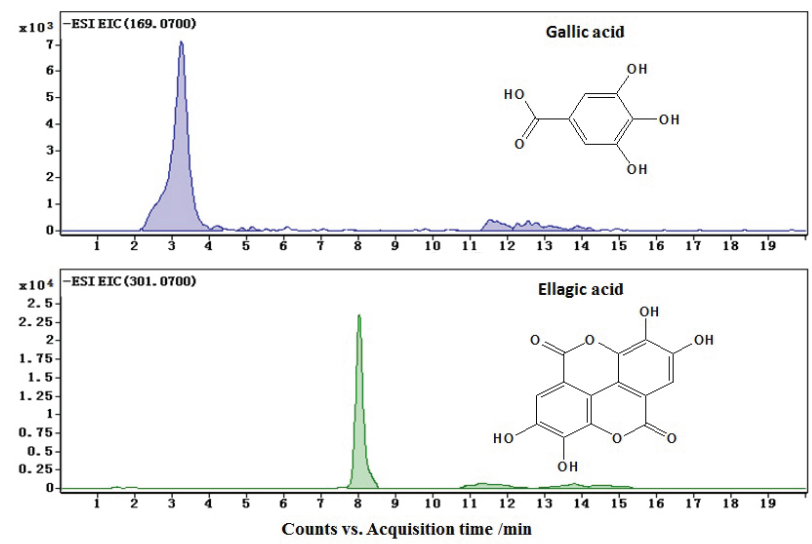

c)
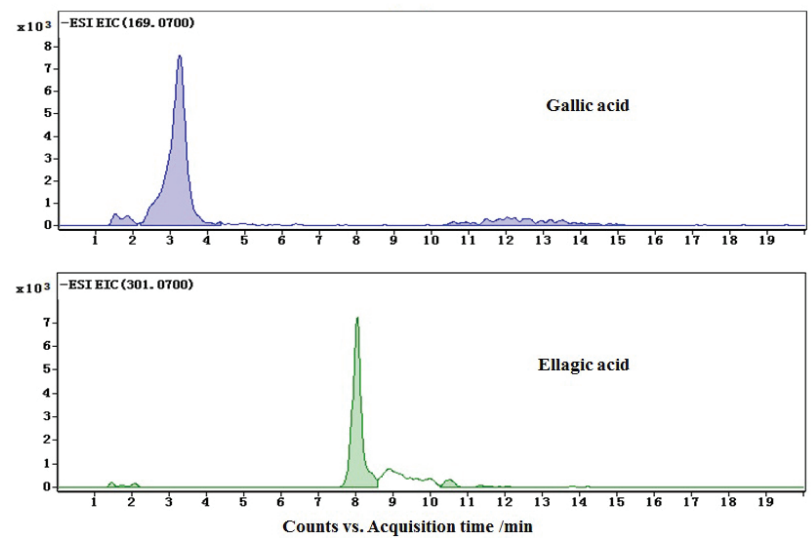

Fig. 1. The chromatograms of ellagic acid and gallic acid: a) blank rat plasma, b) blank rat plasma combined with ellagic acid reference and internal standard solution, c) plasma sample $1 \mathrm{~h}$ after oral administration of $40 \mathrm{mg} \mathrm{kg}^{-1}$ EA-S-SMEDDS. 


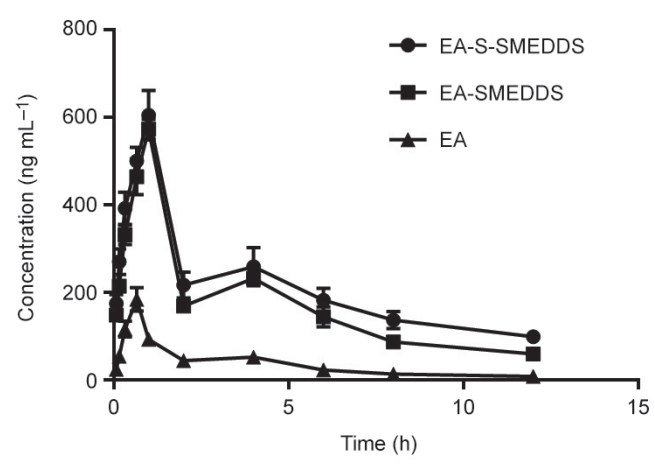

Fig. 2. Mean plasma concentration-time curve of ellagic acid in rats after oral administration of EAS-SMEDDS, EA-SMEDDS and EA suspension at a dose of $40 \mathrm{mg} \mathrm{kg}^{-1}(n=4)$.

Table II. Pharmacokinetic parameters of ellagic acid in rats after oral administration of S-SMEDDS, SMEDDS and the suspension $(n=4, \bar{X} \pm S)$

\begin{tabular}{|c|c|c|c|}
\hline \multirow{2}{*}{ Parameters } & \multicolumn{3}{|c|}{ Formulation } \\
\hline & EA suspension & EA-SMEDDS & EA-S-SMEDDS \\
\hline$A U C_{0-\mathrm{t}}\left(\mu \mathrm{g} \mathrm{mL^{-1 } \mathrm { h } )}\right.$ & $0.436 \pm 0.042$ & $2.079 \pm 0.064^{* *}$ & $2.552 \pm 0.280^{* * \#}$ \\
\hline$A U C_{0-\infty}\left(\mu \mathrm{g} \mathrm{mL} L^{-1} \mathrm{~h}\right)$ & $0.496 \pm 0.028$ & $2.489 \pm 0.239^{* *}$ & $3.340 \pm 0.442^{* * \#}$ \\
\hline $\mathrm{MRT}_{0-\infty}(\mathrm{h})$ & $4.653 \pm 0.605$ & $6.031 \pm 0.900^{*}$ & $7.906 \pm 1.226^{* *}$ \\
\hline$T_{\max }(\mathrm{h})$ & $0.667 \pm 0.000$ & $1.000 \pm 0.000$ & $1.000 \pm 0.000$ \\
\hline$C_{\max }\left(\mu g \mathrm{~mL}^{-1}\right)$ & $0.184 \pm 0.027$ & $0.572 \pm 0.023^{* *}$ & $0.604 \pm 0.057^{* *}$ \\
\hline $\mathrm{CL} / \mathrm{F}\left(\mathrm{L} \mathrm{h}^{-1} \mathrm{~kg}^{-1}\right)$ & $80.820 \pm 4.583$ & $16.180 \pm 1.510^{* *}$ & $12.152 \pm 1.772^{* * \#}$ \\
\hline
\end{tabular}

${ }^{*} p<0.05,{ }^{* *} p<0.01$, statistical significance compared with the EA suspension

${ }^{*} p<0.05$, statistical significance compared with EA-SMEDDS

increase for $C_{\max }$ values compared with EA. The results of the in vivo bioavailability study showed that the peak time of the two emulsions was longer than EA, which might owe to the fact that $T_{\max }$ is the time required for the plasma concentration to reach the maximum value, when the absolute value of 'absorbed dose minus distribution, metabolism and excretion' is maximum. For insoluble drugs, the dissolution process is usually the limiting step of drug absorption, and a higher dissolution rate plays an important role in improving bioavailability (14). In addition, the improved bioavailability of S-SMEDDS could be influenced not only by the quick dissolution but also by the proportion of the dissolution. When S-SMEDDS is emulsified in water, it provides a supersaturation condition and increases the interfacial area for drugs, which helps reduce the precipitation of ellagic acid and further improve the solubility and bioavailability of it (15). And S-SMEDDS could dissolute more completely than the other two groups. Therefore, the oral absorption of ellagic acid in S-SMEDDS was the best among EA suspension, EA-SMEDDS and EA-S-SMEDDS. 


\section{CONCLUSIONS}

A specific and reliable UHPLC-Q-TOF-MS method was established in this study to determine the concentration of ellagic acid in rat plasma with gallic acid as the internal standard. The plasma samples containing ellagic acid held high stability under various conditions. Compared with the EA suspension, EA-S-SMEDDS exhibited a prolonged residence time and higher AUC values in vivo. Therefore, S-SMEDDS could enhance the oral absorption of EA, and the biopharmaceutical advantages of S-SMEDDS indicated promising prospects in the development of EA.

Acknowledgements. - This work was supported by the Key Research and Development Plan Project of Shandong Province (No. 2016GSF202010), Youth Innovation Team of Scientific Research Foundation of the Higher Education Institutions of Shandong Province (No. 2019KJM006), Shandong Provincial Natural Science Foundation (No. ZR2018LH020) and Scientific Research Foundation of the Higher Education Institutions of Shandong Province, China (No. J18K035).

\section{REFERENCES}

1. G. Quan, B. Niu, V. Singh, Y. Zhou, C. Wu, X. Pan and C. Wu, Supersaturable solid self-microemulsifying drug delivery system: precipitation inhibition and bioavailability enhancement, Int. J. Nanomed. 12 (2017) 8801-8811; https://doi.org/10.2147/IJN.S149717

2. N. T. Tung, C. S. Tran, H. A. Nguyen, T. D. Nguyen, S. C. Chi, D. V. Pham, Q. D. Bui and X. H. Ho, Formulation and biopharmaceutical evaluation of supersaturatable self-nanoemulsifying drug delivery systems containing silymarin, Int. J. Pharm. 555 (2019) 63-76; https://doi.org/10.1016/j. ijpharm.2018.11.036

3. Z. Q. Chen, Y. Liu, J. H. Zhao, L. Wang and N. P. Feng, Improved oral bioavailability of poorly watersoluble indirubin by a supersaturatable self-microemulsifying drug delivery system, Int. J. Nanomed. 7 (2012) 1115-1125; https://doi.org/10.2147/IJN.S28761

4. J. M. Landete, Ellagitannins, ellagic acid and their derived metabolites: A review about source, metabolism, functions and health, Food Res. Int. 44 (2011) 1150-1160; https://doi.org/10.1016/j. foodres.2011.04.027

5. F. Lei, D. M. Xing, L. Xiang, Y. N. Zhao, W. Wang, L. J. Zhang and L. J. Du, Pharmacokinetic study of ellagic acid in rat after oral administration of pomegranate leaf extract, J. Chromatogr. B 796 (2003) 189-194; https://doi.org/10.1016/S1570-0232(03)00610-X

6. A. W. R. Hamad, W. M. Al-Momani, S. Janakat and S. A. Oran, Bioavailability of ellagic acid after single dose administration using HPLC, Par. J. Stat. 8 (2009) 1661-1664; https:// DOI: 10.3923/ pjn.2009.1661.1664

7. L. Yan, P. Yin, C. Ma and Y. Liu, Method development and validation for pharmacokinetic and tissue distributions of ellagic acid using ultrahigh performance liquid chromatography-tandem mass spectrometry (UPLC-MS/MS), Molecules 19 (2014) 18923-18935; https://doi:10.3390/molecules191118923

8. J. Wang, D. Zheng, Y. Wang, C. Zhang and X. Sun, Pharmacokinetics study of Erhuang decoction extracts in rats by HPLC-MS/MS, J. Chromatogr. B 1059 (2017) 35-42; https://doi.org/10.1016/j. jchromb.2017.05.019

9. G. R. Valicherla, M. Riyazuddin, S. Shahi, A. P. Gupta, A. A. Syed, A. Husain and J. R. Gayen, LCESI-MS/MS assay development and validation of a novel antidiabetic peptide PSTi8 in mice plasma using SPE: An application to pharmacokinetics, J. Pharm. Biomed. Anal. 180 (2019) 113074; https://doi. org/10.1016/j.jpba.2019.113074 
10. V. Ferrone, M. Carlucci, P. Palumbo and G. Carlucci, Bioanalytical method development for quantification of ulifloxacin, fenbufen and felbinac in rat plasma by solid-phase extraction (SPE) and HPLC with PDA detection, J. Pharm. Biomed. Anal. 123 (2016) 205-212; https://doi.org/10.1016/j. jpba.2016.01.062

11. D. Zheng, C. Lv, X. Sun, J. Wang and Z. Zhao, Preparation of a supersaturatable self-microemulsion as drug delivery system for ellagic acid and evaluation of its antioxidant activities, J. Drug Deliv. Sci. Technol. 53 (2019) 101209; https://doi.org/10.1016/j.jddst.2019.101209

12. G. Z. Pei, W. Chen, H. Zhang and G. H. Li, Pharmacokinetics of ellagic acid tablets in rabbits, Chin. J. Exp. Tradit. Med. Formulae 18(2012) 136-138; https://doi.org/10.13422/j.cnki.syfjx.2012.12.047

13. C. Sun, Y. Gui, R. Hu, J. Chen, B. Wang, Y. Guo, W. Lu, X. Nie, Q. Shen, S. Gao and W. Fang, Preparation and Pharmacokinetics Evaluation of Solid Self-Microemulsifying Drug Delivery System (SSMEDDS) of Osthole, AAPS PharmSciTech 19 (2018) 2301-2310; https://doi.org/10.1208/s12249-0181067-3

14. J. Jinno, N. Kamada, M. Miyake, K. Yamada, T. Mukai, M. Odomi, H. Toguchi, G. G. Liversidge, K. Higaki and T. Kimura, Effect of particle size reduction on dissolution and oral absorption of a poorly water-soluble drug, cilostazol, in beagle dogs, J. Controll. Release 111 (2006) 56-64; https://doi. org/10.1016/j.jconrel.2005.11.013

15. H. Mu, R. Holm and A. Müllertz, Lipid-based formulations for oral administration of poorly watersoluble drugs, Int. J. Pharm. 453 (2013) 215-224; https://doi.org/10.1016/j.ijpharm.2013.03.054 